\title{
Inulanthera brownii (Compositae: Anthemideae): a new combination
}

\section{J. Nicholas Hind ${ }^{1}$}

Summary. A new combination is provided in Inulanthera (I. brownii) to validate a combination proposed by Källersjö in her assessment of the delimitation of Athanasia.

Key Words. Asteraceae, Athanasia, nomenclature.

\section{Introduction}

Current work towards a treatment of the Compositae tribe Anthemideae for Flora Zambesiaca has necessitated a review of the literature and herbarium material, especially for the genus Inulanthera Källersjö. Based on fruit structure, provided a reassessment of the generic delimitation of Athanasia L. In doing so she provided many new combinations in recognising, or rerecognising ('reviving'), several genera; Inulanthera was described as new, and ten new combinations proposed. Regrettably, one of these, a Madagascan endemic, was provided without a basionym reference, and therefore illegitimate as it was published post-1953. In order to provide a valid name for use in any future revision, which is certainly necessary, the following new combination is provided:

Inulanthera brownii (Hochr.) Källersjö ex D. J. N. Hind, comb. nov.

http:/ /www.ipni.org/urn:lsid:ipni.org:names:77136519-1
Athanasia brownii Hochr., Annuaire Conserv. Jard. Bot. Gèneve 11 \& 12: 120 (Hochreutiner 1908). Type: [MADAGASCAR:] 'Imerina, environs de Marmarivo, terrains secs, fleurs jaunes (Rusillon, 1903, n. 54).' holotype $\mathrm{G}(00023878)$; isotype $\mathrm{K}(000410820)$.

Inulanthera brownii (Hochr.) Källersjö, Nordic J. Bot. 5 (6): 540 (1985)[publ. 1986], comb. illeg., without basionym reference.

\section{References}

Hochreutiner, B. P. G. (1908). Sertum Madagascariense: étude systématique de deux collections de plantes récoltées à Madagascar par MM. J. Guillot et $\mathrm{H}$. Rusillon. Accompagnée de conclusions sur la géographie botanique et de clefs analytiques pour plusieurs genres critiques. Annuaire Conserv. Jard. Bot. Gèneve 11 \& 12: 35 - 135.

Källersjö, M. (1985)[publ. 1986]. Fruit structure and generic delimitation of Athanasia (AsteraceaeAnthemideae) and related South African genera. Nordic J. Bot. 5 (6): $527-542$.

Accepted for publication 5 March 2014. Published online 28 March 2014

1 Herbarium, Royal Botanic Gardens, Kew, Richmond, Surrey, TW9 3AB, UK. e-mail: n.hind@kew.org 